\title{
The Gamma Factory proposal for CERN
}

\author{
Mieczyslaw Witold Krasny *† \\ LPNHE, Paris-Sorbonne University and CNRS-IN2P3, Paris, France \\ E-mail: krasnyalpnhe.in2p3.fr
}

\begin{abstract}
This contribution discusses the possibility of broadening the present CERN research programme by a new component making use of a novel concept of the light source. The proposed, Partially Stripped Ion beam driven, light source is the backbone of the Gamma Factory initiative. It could be realised at CERN by using the infrastructure of the already existing accelerators. It could push the intensity limits of the presently operating light-sources by up to 7 orders of magnitude, reaching the flux of the order of $10^{17}$ photons/s, in the particularly interesting $\gamma$-ray energy domain of $1 \leq E_{\text {photon }} \leq 400 \mathrm{MeV}$. This domain is out of reach for the FEL-based light sources based on sub-TeV energy-range electron beams. The unprecedented-intensity, energytuned, quasi-monochromatic gamma beams, together with the gamma-beams-driven secondary beams of polarised positrons, polarised muons, neutrinos, neutrons and radioactive ions would constitute the basic research tools of the proposed Gamma Factory. A broad spectrum of new opportunities, in a vast domain of uncharted fundamental and applied physics territories, could be opened by the Gamma Factory research programme.
\end{abstract}

The European Physical Society Conference on High Energy Physics

5-12 July, 2017

Venice

\footnotetext{
* Speaker.

${ }^{\dagger}$ Gamma Factory group: E.G. Bessonov, Lebedev Physical Institute, Moscow, Russia; D. Budker, Helmholtz Institute, Johannes Gutenberg University, Mainz, Germany; K. Cassou, I. Chaikovska, R. Chehab, K. Dupraz, A. Martens, F. Zomer, LAL Orsay, France; C.Curatolo, L. Serafini Department of Physics, INFN-Milan and University of Milan, Milan, Italy ; O. Dadoun, M. W. Krasny, LPNHE, University Paris VI et VII and CNRS-IN2P3, Paris, France; P. Czodrowski, J. Jowett, Reyes Alemany Fernandez, M. Kowalska, M. Lamont, D. Manglunki, A. Petrenko, F. Zimmermann, CERN, Geneva, Switzerland; W. Placzek, Jagellonian University, Krakow, Poland; Y. K. Wu, FEL Laboratory, Duke University, Durham, USA; M. S. Zolotorev Center for Beam Physics, LBNL, Berkeley, USA.
} 


\section{Existing and future MeV-range light sources}

The light sources in the discussed $\mathrm{MeV}$ energy range have already been constructed and operating in several countries: HI $\gamma$ S-USA, LEPS-Japan, LADON-Italy, ROKK-1-Russia, GRAALFrance and LEGS-USA. The leading future project entering the construction phase is the European Union project ELI-NP. The ELI-NP facility is expected to produce the flux of $10^{13}$ photons/s with the maximum energy of $20 \mathrm{MeV}$. The highest photon flux achieved so far is $10^{10}$ photons/s.

All the above facilities generate, or are expected to generate, the photon beams by the process of the inverse Compton scattering of the laser photons on the highly relativistic electron beams. Since the cross section of the inverse Compton process is small, of the order of $1 \mathrm{barn}$, in order to achieve the quoted fluxes, both the laser system and the energy recovery linac technologies have to be pushed to their technological limits.

\section{The Gamma Factory proposal for CERN - the leap into the ultra-high gamma-source intensity}

The idea underlying the Gamma Factory proposal is to use Partially Stripped Ion (PSI) beams, instead of electron beams, as drivers of the light source ${ }^{1}$. The PSI beams are the beams of ions carrying one, or more, electrons which have not been stripped along the way from the ion source to the final PSI beam storage ring. In the process of the resonant absorption of the laser photons by the PSI beam, followed by a spontaneous atomic-transition emissions of secondary photons, the initial laser-photon frequency is boosted by a factor of up to $4 \times \gamma_{L}^{2}$, where $\gamma_{L}$ is the Lorenz factor of the partially stripped ion beam. Therefore, the light source in the energy range of $1 \leq E_{\text {photon }} \leq 400 \mathrm{MeV}$ could be driven by the high- $\gamma_{L}$, LHC-stored, PSI beams. CERN is a unique place in the world where such a light source could be realized.

The cross-section for the resonant absorption of laser photons by the atomic systems is in the giga-barn range, while the cross-section for the point-like electrons is in the barn range. As a consequence the PSI-beam-driven light source intensity could be higher than those of the electronbeam-driven ones by a large factor. For the light source working in the regime of multiple photon emissions by each of the beam ions, the photon beam intensity is expected to be limited no longer by the laser light intensity but by the available RF power of the ring in which partially stripped ions are stored. For example, the flux of up to $10^{17}$ photons/s could be achieved for photon energies in the $10 \mathrm{MeV}$ region already with the present, $\mathrm{U}=16 \mathrm{MV}$, circumferential voltage of the LHC cavities. This photon flux is by a factor of $10^{7}$ higher than that of the highest-intensity electronbeam-driven light source, HI $\gamma \mathrm{S} @$ Durham, operating in the same energy regime.

Even higher fluxes could be produced if photon beams carrying more than $\mathscr{O}(100 \mathrm{~kW})$ of beam-power can be safely handled, and if the present circumferential voltage could be increased (at LEP2 the corresponding value was $3560 \mathrm{MV}$ ),

\section{Acceleration, storage and use of the PSI beams at CERN}

The first steps to understand the storage stability of the PSI beams were already made at BNL.

\footnotetext{
${ }^{1}$ For the discussion of the light sources based on PSI beams see e.g. [1] and the references quoted therein
} 
The ${ }^{77+} \mathrm{Au}$ beam with two unstripped electrons was successfully circulating in the AGS ring at BNL and, more recently, in its RHIC ring [2]. These tests may be considered as a starting point for further exploratory tests which could be carried out initially at the CERN SPS and, if successful, at the LHC.

If stable PSI beams could be produced and stored they would not only drive the photon source but could also be used for the following two unconventional applications.

Firstly, they would allow the LHC to operate as an electron-proton(ion) collider [3]. The LHC experiments could simply record collisions of electrons, brought to LHC experiment's interaction points "on the shoulders" of the ion-carriers, with the counter-propagating proton(ion) beam.

Secondly, they may turn out to be efficient driver beams for the hadron beam driven plasmawakefield acceleration [4] of a witness beam. This is because the PSI bunches, contrary to the proton bunches, could be very efficiently cooled by the Doppler laser cooling techniques, allowing to compress their bunch sizes. A profit could thus be made from the fact that the maximal achievable plasma electric field acceleration gradient increases quadratically with the decreasing bunch length of the driver beam.

Thirdly, they could provide new possibilities for precision electroweak measurements in hydrogenlike, high-Z atoms for indirect searches of new, Beyond the Standard Model (BSM), effects.

It remains to be stressed that a large fraction of the beam cooling and beam manipulation techniques exploiting the internal degrees of freedom of the beam particles, which have been mastered over the three decades by the atomic physics community, could be directly applied to the high energy PSI beams.

\section{The photon collision schemes and secondary beams of the Gamma Factory}

The high intensity and high brilliance gamma beam could be used to realize, for the first time, a photon-photon collider at CERN: (1) in the range of CM energies of $1-100 \mathrm{KeV}$, for collisions of the gamma beam with the laser photons, and (2) in the energy range of $1-800 \mathrm{MeV}$, for the gamma beam collisions with the counter propagating, twin gamma beam.

The gamma beam could also collide with the LHC proton and fully stripped ion beams. The $\mathrm{CM}$ energy range of the corresponding photon-proton and photon-nucleus colliders would be 4 - $60 \mathrm{GeV}$.

Finally, the gamma beam could be extracted from the LHC and used to produce high intensity secondary beams of:

- Polarized electrons and positrons with the expected intensity which could reach $10^{17}$ positrons/s. Such an intensity would be three orders of magnitude higher than that of the KEK positron source and largely satisfy the source requirements for both the ILC and CLIC colliders, and even that of a future high luminosity ep (eA) collider project based on the energy recovery linac.

- Polarized muon and the tertiary neutrino beams. The intensity of the Gamma Factory polarized muon beams could be sizably higher than that of the Paul Scherrer Institute's " $\pi \mathrm{E} 5 "$ 
muon beam. If accelerated, they could be used to produce high intensity neutrino beams. Thanks to the initial muon polarization the muon-neutrino (muon-anti-neutrino) beams could be uncontaminated by the electron-neutrino (electron-anti-neutrino) contributions. The neutrino and antineutrino bunches could be separated with $100 \%$ efficiency on the bases of their timing. In addition, their fluxes could be predicted to a very high accuracy, providing an optimal neutrino-beam configuration for the high systematic precision measurements e.g. of the $\mathrm{CP}$-violating phase in the neutrino CKM matrix. To reach high muon (neutrino) intensities two paths could be envisaged. In the first one, based on the conversion of the high energy gamma beam into muon pairs, the present circumferential voltage of the LHC would have to be upgraded and a specialized design of the gamma conversion targets would have to be made. An alternative scheme would be to tune the gamma beam energy to a significantly lower energy - just above the electron-positron pair production threshold, reducing thus both the circumferential voltage and the beam power strains. The positron bunches, produced by such a low energy gamma beam, would need to be accelerated in the dedicated positron ring to the energy exceeding the muon pair production threshold in collisions with the stationary target electrons, $E_{e} \sim\left(2 m_{\mu}^{2}\right) /\left(m_{e}\right)$. The intensity of the muon beam produced in such a scheme could be increased by replacing the single-pass collisions of the positron beam by the multipass collisions[5]. For both the above two types of muon beams the product of the beam longitudinal and transverse emittances could be at least four orders of magnitude smaller than that for the pion-decay-originated muon source.

- Neutrons with the expected intensity reaching $10^{15}$ neutrons/s (first generation neutrons) and radioactive, neutron-rich ions with the intensity reaching $10^{14}$ ions/s. Preliminary estimates show that the intensity of the Gamma Factory beams of neutrons and radioactive ions could approach those of the European projects under construction like ESS (and FAIR) and the planned EURISOL facility. The Gamma Factory beams may turn out to be more effective in terms of their power consumption efficiency since almost $10 \%$ of the LHC RF power could be converted into the power of the neutron and radioactive ion beams if the energy of the photon beam is tuned to the Giant Dipole Resonance (GDR) region of the target nuclei.

New Gamma Factory beam lines of unprecedented intensities and its high luminosity photonphoton, photon-proton and photon-nucleus collision interaction points could attract new scientific communities to CERN. This could lead to a diversification of the CERN-based scientific programme.

\section{Expected highlights of the Gamma Factory research programme}

The physics research domains which could be explored by this proposal include: fundamental QED measurements (for example, for the first time ever, the elastic light-light scattering could be observed with the rate of $\approx 1000$ events/s, providing the high-precision QED test); dark matter searches (mainly via the dark photon and neutron portals); investigation of basic symmetries of the Universe (neutron dipole moment, neutron-antineutron oscillations, forbidden muon decays); studies of color confinement; nuclear photonics; physics of neutron-rich radioactive beams, physics 
with energy-tagged neutron beam and the vast domain of the atomic physics of muonic and electronic atoms.

The Gamma Factory's high brilliance beams of polarized positron and muons may help in addressing at CERN the research programme of: (1) a TeV-energy-scale muon collider; (2) neutrino factory, (3) a lepton-hadron collider, and (4) the Deep Inelastic Scattering (DIS) fixed target programme.

The CERN Gamma Factory project could open a wide spectrum of industrial and medical applications in the following domains: muon catalyzed cold fusion; gamma-beam catalyzed hot fusion; Accelerator Driven System (ADS) and Energy Amplifier (EA) research; nondestructive assay and segregation of nuclear waste; transmutation of nuclear waste; material studies of thick objects and production of ions for Positron Emission Tomography (PET) and for the selective cancer-cell therapy with alpha emitters.

\section{The way forward}

The presented above research option may turn out not only to be scientifically attractive but also cost-effective because it proposes to re-use, in a novel manner, the existing CERN accelerator infrastructure. It may be considered as complementary to the present hadron-collision programme and could be performed at any stage of the LHC life-time.

In order to prove that such a future option is not only a conceptually attractive but also a viable one, two initial exploratory paths have been initiated very recently.

The goal of the first one is to perform a detailed validation of the achievable performance figures of the Gamma Factory initiative for each branch of its application domains, to build up the physics case for its research programme and, most importantly, to attract a wide community to this initiative.

The goal of the second one is to prove experimentally the concepts underlying this proposal. Most of the feasibility tests can be performed at the SPS and organised such that the ongoing CERN research programme is barely affected.

\section{References}

[1] E.G. Bessonov, Nucl. Instrum. Meth. B309 (2013) 92-94 and the references quoted therein.

[2] D. Trbojevic, Private communication.

[3] M. W. Krasny, Nucl. Instrum. Meth. A540 (2005) 222.

[4] A. Caldwell, et al., Nature Phys. 5 (2009) 363-367.

[5] M. Antonelli et al., Nucl. Instrum. Meth. A807 (2016) 101-107. 